\title{
ON THE EXISTENCE OF [SCHAUDER] DECOMPOSITIONS IN BANACH SPACES
}

\author{
B. L. SANDERS ${ }^{1}$
}

1. Introduction. Let $\left\{b_{i}\right\}$ be a basis for a linear topological space, and let $\left\{\beta_{i}\right\}$ be the sequence of corresponding "coefficient functionals." Following Arsove and Edwards [1], we refer to $\left\{b_{i}\right\}$ as a Schauder basis provided each $\beta_{i}$ is continuous. If $\left\{b_{i}\right\}$ is a basis for a linear topological space $T$, then each $x \in T$ has a unique representation of the form $x=\sum_{1}^{\infty} x_{i}$ where $x_{i}$ is an element of the one-dimensional subspace spanned by $b_{i}$. Thus it is natural to attempt to generalize the concept of a basis $\left\{b_{i}\right\}$ by replacing the $b_{i}$ 's by subspaces. A sequence $\left\{M_{i}\right\}$ of nontrivial (not necessarily closed) subspaces of a linear topological space $T$ is a decomposition of $T$ provided for each $x \in T$ there exists a unique sequence $\left\{x_{i}\right\}$ such that $x_{i} \in M_{i}$ for all $i$ and $x=\sum_{1}^{\infty} x_{i}$. Associated with a decomposition $\left\{M_{i}\right\}$ is a sequence $\left\{P_{i}\right\}$ of projections (where projection is used in the sense of Taylor [8, pp. 240242]) defined by $P_{j}(x)=x_{j}$ where $x=\sum_{1}^{\infty} x_{i}$ is the unique representation of $x$ in terms of the decomposition $\left\{M_{i}\right\}$. (Note that for each $i$, $M_{i}$ is the range of $P_{i}$, and that $P_{i} P_{j}(x)=0$ if $i \neq j$ and $P_{i} P_{j}(x)=P_{i}(x)$ if $i=j$.) In view of the above terminology for bases, it is natural to call a decomposition a Schauder decomposition provided each of the associated projections is continuous. (The reader is referred to [6] for a brief sketch of the history of these concepts.)

Let $\left\{b_{i}\right\}$ be a basis of a linear topological space $T$ and let $\left\{\beta_{i}\right\}$ be the corresponding sequence of coefficient functionals. For each $i$ let $M_{i}$ be the one-dimensional subspace of $T$ generated by $x_{i}$. Then $\left\{M_{i}\right\}$ is a decomposition of $T$. Further, if $\left\{P_{i}\right\}$ is the sequence of projections associated with $\left\{M_{i}\right\}$, then $P_{i}(x)=\beta_{i}(x) b_{i}$ for all $x \in T$. Clearly $\left\{b_{i}\right\}$ is a Schauder basis of $T$ if and only if $\left\{M_{i}\right\}$ is a Schauder decomposition of $T$. In the second section of this paper we give an example, due to Charles W. McArthur and reproduced here with his permission, of a Schauder decomposition of a nonseparable Banach space. Thus it is seen that the concept of a [Schauder] decomposition is a generalization of the concept of a [Schauder] basis. Although every basis in a Banach space is a Schauder basis, it is not true that

Received by the editors August 4, 1964.

${ }^{1}$ Work supported, in part, by a National Science Foundation Terminal Year Fellowship. The author gratefully acknowledges the guidance of Dr. Charles W. McArthur in the development of this material. The author is indebted to the referee for an improvement of Theorem $(m)$. 
every decomposition of a Banach space is a Schauder decomposition. (See Example 2 in §2.) In $\$ 3$ we discuss the decomposition questions corresponding to the classical basis question of whether every separable Banach space has a basis. In particular it is shown that every Banach space has a decomposition and that certain Banach spaces have Schauder decompositions. The author does not know whether every Banach space has a Schauder decomposition. In fact, the author does not know whether $(m)$, the space of bounded sequences of real numbers with the sup or uniform norm, has a Schauder decomposition. However the following related result is established in $\$ 3$.

THEOREM $(m)$. There does not exist a Schauder decomposition $\left\{M_{p}\right\}$ of $(m)$ for which each of the unit vectors $e_{i}=\left\{\delta_{i j}\right\}_{j=1}^{\infty}(i=1,2, \cdots)$ lies in a distinct subspace $M_{p}$.

\section{Examples.}

EXAMPle 1 (MCARTHUR). A nonseparable Banach space and a Schauder decomposition for it. Let $X$ be a nonseparable Banach space, and let $c_{0}(X)$ be the collection of all sequences in $X$ which converge to zero. The set $c_{0}(X)$ is made into a nonseparable Banach space by using the point-wise definitions of vector addition and scalar multiplication and the sup or uniform norm. For each positive integer $i$ let $M_{i}$ denote the collection of all sequences belonging to $c_{0}(X)$ for which all entries, except possibly the $i$ th one, are zero. One can show that $\left\{M_{i}\right\}$ is a decomposition of $X$ and each $M_{i}$ is closed. Now Theorem 3 in $[5]$ states that in a complete metric linear space, a decomposition is a Schauder decomposition if and only if each of the subspaces in the decomposition is closed. (The necessity is clear [8, Theorem 4.8-C, p. 241]. For a Banach space the sufficiency follows by renorming the space by $\||x|\|=\sup _{n}\left\|\sum_{i=1}^{n} P_{i}(x)\right\|$, using the closedness of the $M_{i}$ 's to establish the completeness of the new space, establishing the continuity of the identity map from the new space into the old, and using the resulting continuity of the identity map from the old space to the new to establish the continuity of the projections.) Hence it is seen that $\left\{M_{i}\right\}$ is a Schauder decomposition of $c_{0}(X)$.

ExAmple 2. A decomposition of a Banach space which is not a $S c h a u d e r$ decomposition. Let $P$ be a projection from $(m)$ on to $\left(c_{0}\right)$, the subspace of $(m)$ consisting of sequences which converge to zero. Let $M_{1}=N(P)$, the null space of $P$, and let $M_{i}$ be the span of $e_{i-1}$ for $i=2,3, \cdots$ Thus $\left\{M_{i}\right\}$ is a decomposition of $(m)$. However, Sobczyk [7] has shown that $P$ is not continuous. Thus, since the range of $P$ is $\left(c_{0}\right)$ and $\left(c_{0}\right)$ is a closed subspace of $(m), M_{1}=N(P)$ is not closed. Thus since the range of the projection, $P_{1}$, associated with 
$M_{1}$, is $M_{1}, P_{1}$ is not continuous [8, Theorem 4.8-C, p. 241], and $\left\{M_{i}\right\}$ is not a Schauder decomposition of $(m)$.

3. Existence theorems. Using the result of Banach [2, p. 238] that every infinite dimensional Banach space $X$ has a closed subspace $X_{1}$ which has a basis (for a proof of this result see Gelbaum [4, p. 29] or Day [3]), one easily proves (using the method of the preceding example with $(m)$ replaced by $X$ and $\left(c_{0}\right)$ replaced by $\left.X_{1}\right)$ the following theorem.

THEOREM 1. Every infinite dimensional Banach space (or normed linear space) has a decomposition.

Although the author does not know whether every infinite dimensional Banach space has a Schauder decomposition, it is easy to obtain the following result.

THEOREM 2. Every separable Banach space which has a subspace which is isometrically isomorphic to $\left(c_{0}\right)$ has a Schauder decomposition.

This result is established by using the method of the preceding example and/or theorem and Sobczyk's result [7, p. 946, paragraph 2] that every separable Banach space having a subspace which is isometrically isomorphic to $\left(c_{0}\right)$ admits a continuous projection onto that subspace.

We turn now to the proof of Theorem $(m)$. First we establish the following lemma.

Lemma. If $\left\{M_{p}\right\}$ is a Schauder decomposition of $(m),\left\{P_{p}\right\}$ is the associated sequence of projections, and $i$ is a one-to-one function of the positive integers into themselves, then the function $P$ defined on $(m)$ by

$$
P(x)=\left\{P_{i(j), j}(x)\right\}_{j=1}^{\infty},
$$

(where $P_{i, k}(x)$ denotes the kth entry of the sequence $P_{i}(x)$ ), is a continuous linear operator from $(m)$ into $\left(c_{0}\right)$.

Proof. We first show that $P(x) \in\left(c_{0}\right)$ for all $x=\left\{x_{i}\right\} \in(m)$. Let $\epsilon>0$ be given. Since $\left\{\sum_{1}^{n} P_{k}(x)\right\}_{n=1}^{\infty}$ converges to $\left\{x_{i}\right\}$ in $(m)$, there exists an $N$ such that for all $n \geqq N$ and for all $m$,

$$
\left|x_{m}-\sum_{k=1}^{n} P_{k, m}(x)\right|<\epsilon / 2 \text {. }
$$

Now for $i(j) \geqq 2$,

$$
\left|P_{i(j), j}(x)\right| \leqq\left|\sum_{k=1}^{i(j)} P_{k, j}(x)-x_{j}\right|+\left|x_{j}-\sum_{k=1}^{i(j)-1} P_{k, j}(x)\right| .
$$


From (1) and (2) it follows that $\left|P_{i(j), j}(x)\right|<\epsilon$ for all sufficiently large $j$. Hence $P(x) \in\left(c_{0}\right)$. The function $P$ is clearly linear, and we need only show that $P$ is continuous. For each $n$, let $U_{n}=\sum_{1}^{n} P_{k}$. Thus $\left\{U_{n}\right\}$ is a sequence of continuous linear operators on $(m)$ such that $\left\{U_{n}(x)\right\}$ converges for each $x \in(m)$. By [2, Theorem 5, p. 80] there exists a real number $K$ such that $\left\|U_{n}\right\| \leqq K$ for all $n$. Hence $\left\|P_{j}\right\| \leqq 2 K$ for all $j$. Thus for each $x \in(m)$ and for each $j,\left|P_{i(j), j}(x)\right|$ $\leqq$ su $\mathrm{p}_{n}\left|P_{i(j), n}(x)\right|=\left\|P_{i(j)}(x)\right\| \leqq 2 K\|x\|$. Hence for each $x \in(m)$, $\|P(x)\| \leqq 2 K\|x\|$, and $P$ is continuous.

Proof of Theorem $(m)$. Assume that $\left\{M_{p}\right\}$ is a Schauder decomposition of $(m)$, and that each of the unit vectors $e_{i}(i=1,2, \cdots)$ lies in a distinct subspace $M_{p}$. Then there exists a one-to-one function $i$ from the positive integers into themselves such that $e_{j} \in M_{i(j)}$ for all $j$. Let $\left\{P_{p}\right\}$ be the sequence of projections associated with $\left\{M_{p}\right\}$. We will show that the operator $P$ defined in the above lemma is a projection from $(m)$ onto $\left(c_{0}\right)$. This will be a contradiction to the result of Sobczyk, referred to in Example 2 above, that there is no continuous projection from $(m)$ onto $\left(c_{0}\right)$, and the theorem will be established. By the above lemma it is sufficient to show that

$$
P(x)=x \text { for all } x \in\left(c_{0}\right) .
$$

Since each $x \in\left(c_{0}\right)$ has a unique representation of the form $x=\sum_{1}^{\infty} a_{k} e_{k}$ and since $P$ is linear and continuous, to establish (1) it is sufficient to show that $P\left(e_{k}\right)=e_{k}$ for each $k=1,2, \cdots$. Since $e_{k} \in M_{i(k)}$, $P_{i(j)}\left(e_{k}\right)=0$ if $i(j) \neq i(k)$ and $P_{i(j)}\left(e_{k}\right)=e_{k}$ if $i(j)=i(k)$. Hence if $j \neq k$, $P_{i(j), j}\left(e_{k}\right)=0$, and if $j=k, P_{i(j), j}\left(e_{k}\right)=1$. Hence $P\left(e_{k}\right)=\left\{P_{i(j), j}\left(e_{k}\right)\right\}_{j=1}^{\infty}$ $=e_{k}$.

\section{BIBLIOGRAPHY}

1. Maynard G. Arsove and R. E. Edwards, Generalized bases in topological linear spaces, Studia Math. 19 (1960), 95-113.

2. S. Banach, Theorie des operations linéaires, 2nd ed. Chelsea, New York, 1955.

3. Mahlon M. Day, On the basis problem in normed spaces, Proc. Amer. Math. Soc. 13 (1962), 655-658.

4. Bernard R. Gelbaum, Notes on Banach spaces and bases, An. Acad. Brasil. Ci. 30 (1958), 29-36.

5. Charles W. McArthur, Infinite direct sums in metric linear spaces. (Unpublished.)

6. B. L. Sanders, Decompositions and reflexivity in Banach spaces, Proc. Amer. Math Soc. 16 (1965), 204-208.

7. Andrew Sobczyk, Projection of the space $(m)$ on its subspace ( $\left.c_{0}\right)$, Bull. Amer. Math. Soc. 47 (1941), 938-947.

8. Angus E. Taylor, Introduction to functional analysis, Wiley, New York, 1961.

Texas Christian University 